\title{
PAPR Analysis of Fifth Generation Multiple Access Waveforms for Advanced Wireless Communication
}

\author{
Kommabatla Mahender ${ }^{1}$, Tipparti Anil Kumar ${ }^{2}$, K.S Ramesh ${ }^{3}$ \\ ${ }^{I}$ Department of Electronics and Communication Engineering, KL University, Vaddeswaram, Andhra Pradesh, India, \\ kmsharma2@yahoo.co.in \\ ${ }^{2}$ Department of Electronics and Communication Engineering, CMR institute of Technology, Kandlakoya, Hyderabad, Telangana, India, \\ tvakumar2000@yahoo.co.in \\ ${ }^{3}$ Department of Electronics and Communication Engineering, KL University, Vaddeswaram, Andhra Pradesh, India, \\ ${ }^{*}$ Corresponding author Email: dr.ramesh@kluniversity.com
}

\begin{abstract}
This paper describes the aspects of multiple access for emerging (5G) Wireless Communication Systems. Orthogonal Frequency Division Multiplexing (OFDM) is best suited for fourth generation (4G) but it suffers from the problem of high Peak to Average Power Ratio (PAPR) \& Side band leakage. Single carrier frequency division multiple access (SC-FDMA) has worked like an alternative to OFDMA only in the uplink process and PAPR was reduced. OFDM based 4G network is not capable of supporting diverse applications and these applications can be implemented by $5 \mathrm{G}$. High traffic requirements of $5 \mathrm{G}$ can be evaluated by using multiple access schemes, namely filter-bank multi-carrier (FBMC), universal-filtered multi-carrier (UFMC), generalized frequency-division multiplexing (GFDM). Comparison of PAPR reduction is done based on Complementary Cumulative Distribution Function (CCDF), for various multiple access $5 \mathrm{G}$ waveforms.
\end{abstract}

Keywords: OFDM, PAPR, SC-FDMA,UFMC, FBMC(Filter-Bank Multi Carrier), GFDM(generalized frequency division multiplexing), $C C D F$ (complementary cumulative distribution function)

\section{Introduction}

Now-a-days in wireless communication the demand for high data rate and quality of services is growing rapidly. The system designers need to adapt techniques that improve the provided QOS and minimizes the effect of delay spread \& Doppler shift. The 4G cellular networks \& LTE, was been optimized to provide high data rate and Band width to strictly synchronized devices like tablets and smart phones using Orthogonal Frequency Division Multiplexing (OFDM) technology. OFDM deals with the multicarrier modulation and can provide high data rate transmission along with bandwidth efficiency .It also combat multi-path fading with low implementation complexity.

Although it has efficient implementation and robustness to channel delays, OFDM suffers from several drawbacks like high PAPR and high out of band sidelobes. High PAPR increases the non-linear behavior of Power Amplifier thereby increases BER and in-band distortion.High out of band side lobes reduces the spectral efficiency of OFDM.OFDM also cause time and frequency offsets and to overcome this it add Cyclic Prefix $(\mathrm{CP})$ to message signal for synchronizing users but at the cost of spectral efficiency.

To overcome some of the above mentioned drawbacks in OFDM,5G communication introduces new transmission schemes. Similar to $4 \mathrm{G}$,these new transmission schemes support huge number of users with high data rates but with low latency.This communication is also spectrally efficient by avoiding frequency and time offsets .
The concept of Internet-of-Things (IoT) is based on machine-tomachine communications with high data rates and heavy traffic with huge number of terminals therefore in near future $5 \mathrm{G}$ interface communication has to meet new requirements. This paper deeply investigates on most promising waveform candidates which are filtered versions of OFDM and signal is filtered on the basis of sub-band or sub-carrier.The transmitted signals are filtered on the basis of sub-carrier using GFDM and FBMC .

FBMC is used to reduce signal sidelobes by using long frequency selective filters compared to OFDM,FBMC is a multicarrier technique which use per-subcarrier filtering. Anyway, by providing additional filtering in FBMC increases the implementation complexity and make it disadvantageous for short burst uplink communication .

Universal filtered multi-carrier is generalization of OFDM and FBMC modulation schemes. OFDM employs the total band filtration and in FBMC individual subcarriers are filtered .In UFMC a group of subcarriers (sub bands) are filtered and it reduces the filter length (when compared with FBMC) and provides sidelobe attenuation. UFMC uses a IFFT(full size inverse fast Fourier transform) to generate each sub-band before initially filtering the time-domain signal using band pass filters. UFMC improves protection of inter symbol interference (ISI) by using special or unified stucture of the frames and therefore it does not have to use a cyclic prefix.

In GFDM, filtering operation is done using a cyclic convolution, thereby avoiding filter tails and therefore GFDM is more suitable for short bursts 


\section{Candidate Air Interfaces}

This section explains about 5G candidate waveforms for broadband communications and also explains about the operating principles based on transceiver schemes

\subsection{Filter Bank Multicarrier Transceiver}

As shown in fig 1 ,it is used to explain operation of FBMC. It utilizes a prototype filter $\mathrm{h}(\mathrm{n})$ of length $\mathrm{KN}$ and using this it resolves the spectral containment complication of OFDM.The number of sub-carrier used is $\mathrm{N}$ and $\mathrm{K}$ is known as Overlapping filter.In time domain analysis of FBMC each symbol will overlap with neighboring $\mathrm{K}$ symbols.Using Nyquist criterion,Inter-Symbol interfeotrence is eliminated. In frequency domain Nyquist filter is designed by $2 \mathrm{~K}-1$ symmetric samples[22].The full Nyquist filter is divided into two similar square root nyquist filters at transmitter and receiver.These two filters are prototype filters and the corresponding prototype filter $\mathrm{h}(\mathrm{n})$ is generated by using $\mathrm{KN}$-point IFFT in frequency domain with a roll -off $\beta=1$. At the transmitter FBMC signal is generated as follows[23].OQAM symbols $s_{k}(n)$ are generated from $\mathrm{QAM}$ symbols $d_{k}(l)$ for a $\mathrm{k}^{\text {th }}$ subcarrier as

for $k$ even, $\left\{\begin{aligned} s_{k}(n) & =\operatorname{Re}\left[d_{k}(l)\right] \\ s_{k}(n+1) & =\operatorname{Im} \operatorname{Re}\left[d_{k}(l)\right] j\end{aligned}\right.$

for $k$ odd, $\left\{\begin{array}{l}s_{k}(n)=\operatorname{ImRe}\left[d_{k}(l)\right] j \\ s_{k}(n+1)=\operatorname{Re}\left[d_{k}(l)\right]\end{array}\right.$

where $n=2 l$ and thus OQAM stream is defined at double the QAM symbol rate. Transmission filter $f k(m)$ is used for convolution, after up-sampling the stream $s_{k}(n)$ ) by a factor $\mathrm{N} / 2$. For a $\mathrm{k}^{\text {th }}$ subcarrier

$f_{k}(m)=h(m) e^{j 2 \pi \frac{k m}{N}}, m=0,1, \ldots, K N-1$

At the transmitter FBMC baseband signal $\mathrm{x}(\mathrm{m})$ is generated by the addition of the filtered stream of each sub-carrier:

$\mathrm{x}(\mathrm{m})=\sum_{N-1}^{N-1} \sum_{+\infty}^{n+\infty} s_{k}(n) f_{k}\left(m-\frac{n N}{2}\right)$

Assuming a perfect noiseless transmission channel, the received symbol at a given sub-channel index $k_{0}$ and time index $n_{0}$ is

$\hat{s_{k 0}}(n 0)=\sum_{N-1}^{N-1} \sum_{+\infty}^{n+\infty} t_{k 0-k, n 0-n} s_{k}(n)$

where $t_{k 0-k, n 0-n}$ is the transmultiplexer response. At the receiver filter $g k(m)$ is matched with the corresponding transmission filter $f k(m)$. From the analysis of transmultiplexer we make a conclusion that the process of filtering eliminates orthogonality between sub-carriers.At transceiver for even time imaginary part of impulse response crosses zero when the real part crosses zero for odd indexes.Thus from (1) OQAM process restores orthogonality for any specific sub-carrier since it generates alternating imaginary and real parts of QAM symbols and also alternates them between sub-carriers at same instant .At the receiver the down-sampling is performed by N/2 to maintain the same throughput.At the receiver OQAM demodulation is performed to generate the estimated QAM symbols $\wedge d_{k}(l)$.FIR equalizer is used at the receiver, to compensate multipath interference, before OQAM demodulation. Usage of OQAM makes FBMC not suitable for MIMO techniques.This technique is also not used for short bursts due to long filter tail.

\subsection{GFDM}

The working convention of the GFDM transmitter and receiver is explained in Fig. 2.In this technique each subcarrier is filtered separately similar to FBMC.GFDM introduces time dimension in data blocks along with the frequency

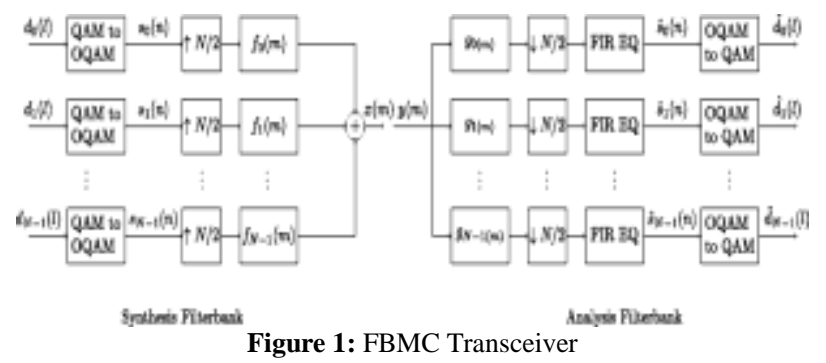

dimension.A GFDM generates a symbol which contains M QAM symbols for each of $\mathrm{N}$ sub-carriers.therefore GFDM is similar as Single carrier system with equalization in frequency domain. A circular filter is used for each sub-carrier in GFDM and this technique avoids the long filter tails as in FBMC,The sub-carrier circular filter is defined as:

$\tilde{f}(m)=f\left(\left(m+\frac{M N}{2} \bmod M N\right)-M N / 2\right)$

where $\tilde{f}(m)$ is a root-raised-Cosine (RRC) filter and $M N$ is filter length.GFDM symbols $\mathrm{N}$ are up-sampled and spanning is implemented with roll-off $\beta<1$. The signal is unchanged before and after filtering due to circular filtering or tail biting.Thus at the transmitter the GFDM generates a baseband signal expressed as:

$\mathrm{x}(\mathrm{m})=\sum_{l=0}^{M-1} \sum_{k=0}^{N-1} d_{k}(l) \tilde{f}[m-l N] \mathrm{e}^{j 2 \pi \frac{\mathrm{km}}{\mathrm{N}}}$

For a $k^{\text {th }}$ subcarrier set of $M$ QAM symbols is given by $d_{k}(l)$ and sample index $m=0, \ldots, N M-1$.

At the receiver a $\mathrm{CP}$ is inserted in the signal to enable single-tap FDE.At receiver filtering of each sub-carrier demonstrates intercarrier-interference(ICI).This interference is handled at receiver by demodulation methods[5] namely matched filter, zero-forcing and (MMSE) minimum mean square error. To trade-off between computational complexity and BER performance [5], this paper implements matched filter receiver along with interference cancellation algorithm. The receiver of GFDM implements same RRC filter as at the transmitter for filter matching, i.e. $\tilde{g}(m))=$ $\tilde{f}(m)$.

Interference appears due to neighbouring sub-carriers and hence suppressed by using a double-sided serial interference cancellation (DSIC) scheme. DSIC is an iterative scheme which estimates the interference $z^{(i)}(m)$ for each sub-carrier and this is retrieved to the received signal $y(m)$.After completion of iterations this scheme cleans all the sub-carriers and or every sub-iteration cleaning of each sub-carrier is implemented and is denoted by index $i$.The expected interference for $i^{\text {th }}$ sub-iteration for $k^{\text {th }}$ sub-carrier is given by

$z^{(i)}(m)=\sum_{k^{\prime}=\left\{\begin{array}{c}k-1,\} \\ k+1\end{array}\right\}} \sum_{n=0}^{M-1} \hat{d}_{k^{\prime}}^{(i)}(l) \tilde{g}(m-l N) \mathrm{e}^{-j 2 \pi \frac{k^{\prime} m}{N}}$

where estimated symbols $\hat{d}_{k^{\prime}}^{(i)}(l)$ are obtained by mapping of received symbols $\hat{d}_{k^{\prime}}^{(i)}(l)$ to the constellation grid. Using the most new estimated data symbols, $(k+1)^{\text {th }}$ sub-carrier is cleaned. BER of IC algorithm approaches to OFDM when simulated for $J=4$ full iterations and gain is unaltered after four iterations.This scheme cannot be applied directly for MIMO techniques. In tail biting scheme Spectral containment is degraded due to severe discontinuities between successive blocks . As stated in [16], to minimize out-of-band radiations.a $M N$-point RRC window is applied for every GFDM block after inserting cyclic prefix. At the receiver robustness of multi-path channels is maintained by dropping last and first time slots of GFDM block(i.e. GS $=2$ guard symbols is inserted) by avoiding windowing compensation. 


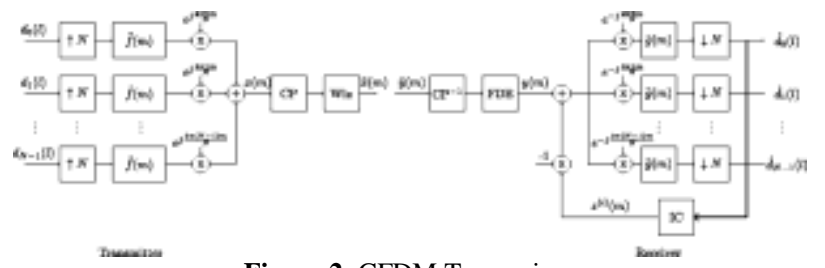

Figure 2: GFDM Transceiver

\subsection{Universal Filtered Multi Carrier}

The principle of operation of UFMC transceiver can be explained by figure 3.The signal is filtered in this on a sub-band basis. The total bandwidth is divided into $N$ sub-carriers and each sub-carrier is subdivided into $B$ sub-bands and orthogonality is maintained thereby avoiding OQAM scheme.UFMC allows reuse of legacy MIMO techniques.Each sub-band $i$ is filtered by DolphChebyshev prototype filter fi $(\mathrm{m})$ which is modulated around center frequency of the sub-band with a filter length $\mathrm{L}_{\mathrm{UFMC}}$ and a side lobe attenuation $\alpha . d_{i}(l)$ undergoes N-point IFFT and parallel to serial conversion to generate time -domain signal $s_{i}(n)$ of $\mathrm{i}^{\text {th }}$ sub-band.$d_{i}(l)$ vector is cluster of QAM images stacking $\mathrm{i}^{\text {th }}$ subband at time $l[15]$. The discrete baseband UFMC flag is gotten for each square of N QAM images by including all filtered signals of each sub-band

$\mathrm{x}(\mathrm{m})=\sum_{i=0}^{B-1} \sum_{n=0}^{L-1} s_{i}(n) f_{i}(m-n)$

where $m=0, \ldots ., N+L_{\mathrm{UFMC}}-1$ samples.Each UFMC block introduces a guard interval of length $L_{\mathrm{UFMC}}-1$ with zero padding. The time dispersion obtained from filters is compensated by guard interval.In severe multipath channel the time dispersion cannot be mitigated[27] if extra guard intervals in UFMC blocks is not introduced[15],[[16].However,for a block span $N+L_{\mathrm{UFMC}}+\mathrm{ZP}_{L}$ -1 samples, this paper proposes introduction of guard interval of length $\mathrm{ZP}_{\mathrm{L}}$ with zero padding(ZP) .Using a simple 1-tap FDE channel time dispersion is mitigated.

UFMC receiver converts received serial data to parallel form and then a $2 \mathrm{~N}$-point FFT is applied on each UFMC symbol .All the $\mathrm{N}$ even sub-carriers are used for retrieve data since ICI is contained in all odd sub-carriers[26]. Final recovery of data symbols is obtained using 1-tap FDE.

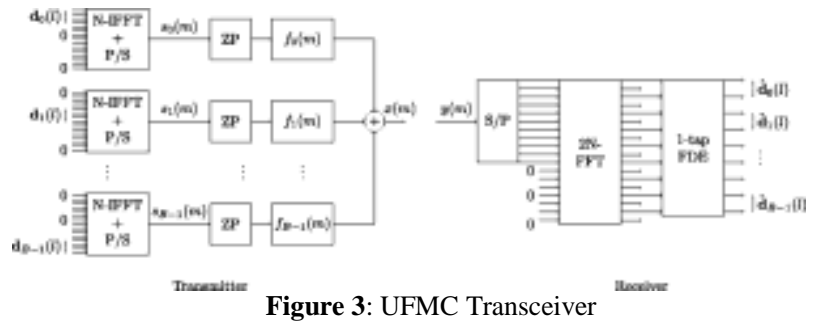

\section{Performance Comparison of Simulation Results}

Sensitivity of each candidate waveform to the non-linear behavior of power amplifier is obtained by the PAPR CCDF curves depicted in fig 4.These curves were obtained by conducting a simulation of around 100,000 multi-carrier symbols. The behavior of all candidates is very close to OFDM since all are multicarrier with equal number of sub-channels.From the simulation results we analyse that UFMC has a slightly higher PAPR than other waveforms.From simulations we also conclude that reduction of sub-carriers result in reduction of spectral containment and reduces spectral efficiency.In this paper we prove that GFDM is attractive with high number of sub-carriers.

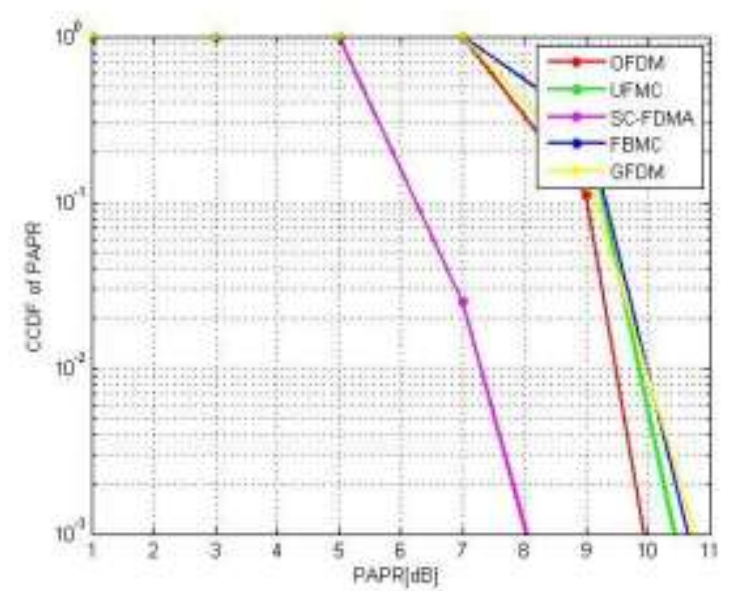

Figure 4: CCDF of Peak to Average Power Ratio

\section{Conclusion}

In this paper we have studied about several limitations of OFDM and how it can be improved with different types of candidate waveforms.These new waveforms were compared and studied based on future requirement of wireless communication.These waveforms were flexible in the design constraints of transmitter and receiver. The narrow bandwidth of FBMC make the prototype filter length greater than that of symbols and make it unsuitable for short burst applications.UFMC filter is relatively shorter compared to FBMC.Time synchronization sensitivity is more in UFMC.The non-orthogonality of GFDM provides pulse shaped subcarriers resulting in self created interference.

However an interference cancellation scheme at the receiver can improve the performance.Thus these new candidate waveforms in different schemes have the potential to provide future requirements of $5 \mathrm{G}$ replacing $\mathrm{OFDM}$.

\section{References}

[1] G Wunder, M Kasparick, S ten Brink, F Schaich, T Wild, I Gaspar, E Ohlmer, SKrone, N Michailow, A Navarro, G Fettweis, D Ktenas, V Berg, M Dryjanski, SPietrzyk, B Eged, in IEEE 77th Vehicular Technology Conference. 5GNOW:Challenging the LTE Design Paradigms of Orthogonality andSynchronicity, (2013), pp 1-5. doi:10.1109/VTCSpring.2013.6691814

[2] G Andrews, S Buzzi, W Choi, SV Hanly, A Lozano, ACK Soong, JC Zhang, What will 5G be? IEEE J. Sel. Areas Commun. 32(6), 1065-1082 (2014). doi:10.1109/JSAC.2014.2328098

[3] Z Wang, GB Giannakis, Wireless Multicarrier Communications. IEEE Signal Process Mag. 17(3), 29-48 (2000). doi:10.1109/79.841722

[4] MG Bellanger, FBMC physical layer: a primer. Technical report, PHYDYAS (2010). http://www.ictphydyas.org/teamspace/internal-folder/FBMCPrimer_ 06-2010.pdf. Accessed 4 Oct 2016

[5] N Michailow, R Datta, S Krone, M Lentmaier, G Fettweis, in German Microwave Conference (GeMiC). Generalized Frequency Division

[6] Multiplexing: A Flexible Multi-Carrier Modulation Scheme for $5^{\text {th }}$ Generation Cellular Networks, (2012). https://mns.ifn.et.tudresden.de/ Lists/nPublications/Attachments/809/main.pdf

[7] T Wild, F Schaich, Y Chen, in 19th International Conference on Digital Signal Processing. 5G Air Interface Design Based on Universal Filtered (UF-)OFDM, (2014), pp. 699-704 doi:10.1109/ICDSP.2014.6900754

[8] J Li, E Bala, R Yang, Resource Block Filtered-OFDM for Future Spectrally Agile and Power Efficient Systems. Phys. Commun. 11, 36-55 (2014). doi:10.1016/j.phycom.2013.10.003

[9] M Matthe, LL Mendes, I Gaspar, N Michailow, D Zhang, G Fettweis, Multiuser time-reversal stc-gfdma for future wireless networks. EURASIP J. Wirel. Commun. Netw. 2015(1), 132 (2015). doi:10.1186/s13638-015-0366-6 
[10] SA Cheema, K Naskovska, M Attar, B Zafar, M Haardt, in WSA 2016; $20^{\text {th }}$ International ITG Workshop on Smart Antennas. Performance comparison of space time block codes for different $5 \mathrm{G}$ air interface proposals, (Munich, 2016), pp. 1-7gfdm, (2015), pp. 1-2. doi:10.1109/VTCFall.2015.7391033

[11] M Renfors, T Ihalainen, TH Stitz, in 2010 European Wireless Conference (EW). A block-Alamouti scheme for filter bank based multicarrier transmission, (2010), pp. 1031-1037. doi:10.1109/EW.2010.5483517

[12] R Zakaria, DL Ruyet, M Bellanger, in 2010 European Wireless Conference (EW). Maximum likelihood detection in spatial multiplexing with fbmc, (2010), pp. 1038-1041. doi:10.1109/EW.2010.5483520

[13] B Farhang-Boroujeny, OFDM Versus Filter Bank Multicarrier IEEE Signal Process Mag. 28(3), 92-112 (2011). doi:10.1109/MSP.2011.940267

[14] T Fusco, A Petrella, M Tanda, in 3rd International Symposium on Communications on Communications, Control and Signal Processing.

[15] Sensitivity of Multi-User Filter-Bank Multicarrier Systems to Synchronization Errors, (2008), pp. 393-398. doi:10.1109/ISCCSP.2008.4537257

[16] F Schaich, T Wild, Y Chen, in 79th IEEE Vehicular Technology Conference. Waveform Contenders for $5 \mathrm{G}$ - Suitability for Short Packet and Low Latency Transmissions, (2014), pp. 1-5. doi:10.1109/VTCSpring.2014.7023145

[17] M Kasparick, Y Chen, J-B Doré, M Dryjanski, IS Gaspar, 5G Waveform Candidate Selection D 3.2. Technical report, 5GNow (2014). http://www. 5gnow.eu/wpcontent/uploads/2015/04/5GNOW_D3.2_final.pdf. Accessed 30 Dec 2016

[18] I Gaspar, N Michailow, A Navarro, E Ohlmer, S Krone, G Fettweis, in $77^{\text {th }}$ IEEE Vehicular Technology Conference. Low Complexity GFDM Receiver Based on Sparse Frequency Domain Processing, (2013), pp. 1-6. doi:10.1109/VTCSpring.2013.6692619

[19] A Aminjavaheri, A Farhang, A RezazadehReyhani, B FarhangBoroujeny, in IEEE Signal Processing and Signal Processing Education Workshop. Impact of timing and frequency offsets on multicarrier waveform candidates for 5G, (2015), pp. 178-183. doi:10.1109/DSP-SPE.2015.736954

[20] R Gerzaguet, D Kténas, N Cassiau, J-B Doré, Comparative study of $5 \mathrm{G}$ waveform candidates for below $6 \mathrm{GHz}$ air interface. Technical report,LETI, CEA Tech (2016). https://docbox.etsi.org/Workshop/2016/ 201601_FUTURERADIOTECHNOL_WORKSHOP/ S05_NEW_RADIO_ACCESS_TECHNO_SERV_ENVIR_PART_ $2 /$ 5G_WAVEFORM_COMPARATIVE_STUDY_BELOW_6GHZ_ KTENAS_CEA_LETI. pdf

[21] X Zhang, L Chen, J Qiu, J Abdoli, On the waveform for 5G. IEEE Commun. Mag. 54(11), 74-80 (2016) doi:10.1109/MCOM.2016.1600337CM

[22] YS Cho, J Kim, WY Yang, CG Kang, MIMO-OFDMWireless Communications with MATLAB_. (Wiley, 2010). doi:10.1002/9780470825631.refs http://dx. doi.org/10.1002/9780470825631.refs

[23] MG Bellanger, in IEEE International Conference on Acoustics, Speech, and Signal Processing. Specification and design of a prototype filter for filter bank based multicarrier transmission, vol. 4, (2001), pp. 2417-2420. doi:10.1109/ICASSP.2001.940488

[24] M Payaró, A Pascual-Iserte, M Nájar, in European Wireless Conference. Performance comparison between FBMC and OFDM in MIMO systems under channel uncertainty, (2010), pp. 1023 1030. doi:10.1109/EW.2010.548352

[25] ] S Van Caekenberghe, S Pollin, A Bourdoux, L Van der Perre, J Louveaux, in 32nd WIC Symposium on Information Theory in the Benelux. Preamble-Based Channel Estimation for Filterbank Multicarrier Wireless Systems, (Brussels, 2010)

[26] J Louveaux, L Baltar, D Waldhauser, M Renfors, M Tanda, C Bader, E Kofidis, PHYDYAS D 3.1. Technical report, PHYDYAS (2008). www.ictphydyas.org/ delivrables/PHYDYASD3.1.pdf/at_download/file. Accessed 20 Sept 2016

[27] X Wang, T Wild, F Schaich, A Fonseca dos Santos, in 20th European Wireless Conference. Universal Filtered Multi-Carrier with Leakage-Based Filter Optimization, (Barcelona, 2014), pp. 15

[28] X Wang, Channel Estimation and Equalization for 5G Wireless Communication Systems Master's thesis. Institut für Nachrichten übertragung, Universität Stuttgart (2014)
[29] SS Prasad, CK Shukla, RF Chisab, in Third International Conference on Computing Communication Networking Technologies. Performance analysis of OFDMA in LTE, (2012), pp. 1-7. doi:10.1109/ICCCNT.2012.6395933

[30] F Horlin, A Bourdoux, Digital Compensation for Analog FrontEnds: a New Approach toWireless Transceiver Design. (Wiley, Chichester, 2008)

[31] A Maltsev, A Lomayev, A Khoryaev, A Sevastyanov, R Maslennikov, in $7^{\text {th }}$ IEEE Consumer Communications and Networking Conference. Comparison of Power Amplifier NonLinearity Impact on $60 \mathrm{GHz}$ Single Carrier and OFDM Systems, (2010), pp. 1-5. doi:10.1109/CCNC.2010.5421601

[32] M Webster, K Halford, Suggested PA Model for $802.11 \mathrm{HRb}$ Technical report, Intersil Corporation (2000) http://www.ieee802.org/11/Documents/DocumentArchives/2000_d ocs/02948Sb-Suggested $\% 20 \mathrm{PA}$

\%20Model\%20for\%20802.11\%20HRb.ppt. Accessed 20 Sept 2016

[33] G Fettweis, M Krondorf, S Bittner, in 69th IEEE Vehicular Technology Conference. GFDM - Generalized Frequency Division Multiplexing, (2009), pp. 1-4. doi:10.1109/VETECS.2009.5073571

[34] [H Bouhadda, H Shaiek, D Roviras, R Zayani, Y Medjahdi, R Bouallegue, Theoretical analysis of BER performance of nonlinearly amplified FBMC/OQAM and OFDM signals. EURASIP J. Adv. Signal Process. 2014(1), 1-16 (2014). doi:10.1186/1687-6180-2014-60

[35] N Michailow, I Gaspar, S Krone, M Lentmaier, G Fettweis, in International Symposium on Wireless Communication Systems. Generalized frequency division multiplexing: analysis of an alternative multi-carrier technique for next generation cellular systems, (2012), pp. 171-175. doi:10.1109/ISWCS.2012.6328352

[36] $T$ Wild, $F$ Schaich, in IEEE 81st Vehicular Technology Conference. A Reduced Complexity Transmitter for UF-OFDM, (2015), pp. 1-6. doi:10.1109/VTCSpring.2015.7145643 